PROCEEDINGS OF THE

AMERICAN MATHEMATICAL SOCIETY

Volume 131, Number 10, Pages 3285-3288

S 0002-9939(03)06888-6

Article electronically published on February 14, 2003

\title{
HIGHER DIMENSIONAL APOSYNDETIC DECOMPOSITIONS
}

\author{
JAMES T. ROGERS, JR.
}

(Communicated by Ronald A. Fintushel)

\begin{abstract}
Let $X$ be a homogeneous, decomposable continuum that is not aposyndetic. The Aposyndetic Decomposition Theorem yields a cell-like decomposition of $X$ into homogeneous continua with quotient space $Y$ being an aposyndetic, homogeneous continuum.

Assume the dimension of $X$ is greater than one. About 20 years ago the author asked the following questions:

Can this aposyndetic decomposition raise dimension? Can it lower dimension? We answer these questions by proving the following theorem.

Theorem. The dimension of the quotient space $Y$ is one.
\end{abstract}

\section{INTRODUCTION}

The Aposyndetic Decomposition Theorem [J1] of F. Burton Jones is essential to the study of homogeneous continua. It goes like this.

Theorem 1. If $X$ is a homogeneous, decomposable continuum that is not aposyndetic, then $X$ admits a continuous decomposition into mutually homeomorphic, indecomposable, homogeneous continua such that the quotient space $Y$ is an aposyndetic, homogeneous continuum.

The author has strengthened this result by showing that (1) the elements of this decomposition must be cell-like continua [R1], and (2) the elements of this decomposition have the same dimension as $X[\mathrm{R} 6]$.

In 1983 the author wrote a survey paper [R4] exposing the state of the art in the study of homogeneous continua. He raised a number of questions, about half of which have been answered. One of the unanswered ones is the following R4 Question 11, p. 224]:

Question 2. Can this aposyndetic decomposition raise dimension? lower dimension?

In this paper we answer this question by proving the following theorem:

Theorem. If $X$ is a homogeneous, decomposable continuum that is not aposyndetic, then the dimension of the quotient space $Y$ of the aposyndetic decomposition of $X$ is one.

Received by the editors July 19, 2001 and, in revised form, May 9, 2002.

2000 Mathematics Subject Classification. Primary 54F15; Secondary 54F50.

Key words and phrases. Continuum, homogeneous, aposyndetic decomposition, terminal subcontinuum, cell-like. 


\section{RESULTS}

A continuum is a compact, connected, nonvoid metric space. A continuum is indecomposable if it is not the union of two of its proper subcontinua. A continuum is hereditarily indecomposable if each of its subcontinua is indecomposable. R. H. Bing [B] has constructed hereditarily indecomposable continua of dimension $n$ for $1 \leq n \leq \infty$.

Let $x$ and $y$ be points of the continuum $X$. If $X$ contains an open set $G$ and a continuum $H$ such that $x \in G \subset H \subset X-\{y\}$, then $X$ is aposyndetic at $x$ with respect to $y$. If $X$ is aposyndetic at each of its points with respect to every other point, then $X$ is aposyndetic.

A continuum $X$ is cell-like if each map of $X$ into a polyhedron is homotopic to a constant map. A continuum is tree-like if it is cell-like and one-dimensional.

A continuum $X$ is homogeneous if for each pair of points $p$ and $q$ belonging to $X$, there exists a homeomorphism $h: X \rightarrow X$ such that $h(p)=q$. The author R5. has shown that each homogeneous, hereditarily indecomposable, nondegenerate continuum is tree-like, and hence one-dimensional.

A subcontinuum $Z$ of the continuum $X$ is terminal if each subcontinuum $W$ of $X$ that intersects $Z$ satisfies either $W \subset Z$ or $Z \subset W$. For example, if $X$ is the topologist's $\sin 1 / x$ curve and $Z$ is the "limit bar," then $Z$ is a terminal subcontinuum of $X$.

A decomposition of $X$ into continua is terminal if each element of the decomposition is a terminal subcontinuum of $X$. Jones [J2] has shown that his aposyndetic decomposition is terminal.

If $f: X \rightarrow Y$ is a map and $y$ is a point of $Y$, then the set $f^{-1}(y)$ is a fiber of $f$. If each fiber of the map $f$ is a cell-like continuum, then $f$ is a cell-like map.

We use reduced Čech cohomology with integral coefficients. A space is acyclic if each of its cohomology groups is trivial. Note that a cell-like continuum is acyclic.

A nondegenerate continuum $Y$ has cohomological dimension one if $H^{q}(Y, B)=0$ for every closed subset $B$ of $Y$ and for every $q>1$. It is known that a continuum is one-dimensional if and only if it has cohomological dimension one [W] p. 109].

Theorem 3. Let $X$ be a decomposable, homogeneous continuum that is not aposyndetic, and let $f: X \rightarrow Y$ be the quotient map of the aposyndetic decomposition of $X$. Then $\operatorname{dim} Y=1$.

Proof. Since $X$ is decomposable, $Y$ is a nondegenerate continuum. Hence $\operatorname{dim} Y \geq$ 1. If $\operatorname{dim} X=1$, then $Y$, being the cell-like image of $X$, is also one-dimensional $\underline{\mathrm{W}}$ p. 113].

Suppose $\operatorname{dim} X>1$. Each fiber $f^{-1}(y)$ is a homogeneous continuum with the property that $\operatorname{dim} f^{-1}(y)=\operatorname{dim} X[\mathrm{R} 6$. Homogeneous, hereditarily indecomposable continua have dimension less than or equal to one [R5], and fibers of $f$ are homogeneous continua, so no fiber of $f$ is hereditarily indecomposable. Since the decomposition is terminal, each hereditarily indecomposable subcontinuum of $X$ is contained in a fiber of $f$.

M. Levin [L] and J. Krasinkiewicz [Kra] have shown that there exists a map $p: X \rightarrow I$ of $X$ onto the unit interval $I$ such that each component of each fiber $p^{-1}(t)$ is hereditarily indecomposable. Let $g: X \rightarrow Z$ and $h: Z \rightarrow I$ be the monotone-light factorization [N, p. 279] of $p$. Each fiber $g^{-1}(z)$ is a hereditarily indecomposable continuum (possibly a point), so each fiber of $g$ is contained in 
a fiber of $f$. It follows that there exists a monotone map $k: Z \rightarrow Y$ satisfying $f=k \circ g$.

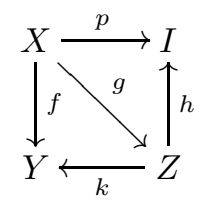

Each fiber of $h$ is totally disconnected, so $Z$ is one-dimensional [HW, Theorem VI7, p. 91].

It suffices to show that the cohomological dimension of $Y$ is one. Let $B$ be a closed subset of $Y$. Consider $H^{q}(Y, B)$ for $q>1$. Let $A=f^{-1}(B)$ and $C=k^{-1}(B)$. Since each fiber of $f$ is cell-like, each fiber has trivial cohomology. The Vietoris-Begle Theorem [S, p. 344] implies that $f^{*}: H^{q}(Y, B) \rightarrow H^{q}(X, A)$ is an isomorphism. Since $f=k \circ g$, we have $f^{*}=g^{*} \circ k^{*}$. Since $Z$ is one-dimensional, $H^{q}(Z, C)=0$. Hence $H^{q}(Y, B)=0$ as well. This completes the proof.

As a consequence of this theorem, we obtain some information on the cohomology groups of homogeneous, decomposable continua that are not aposyndetic. First we need a definition.

A continuum $X$ is unicoherent if each pair of subcontinua of $X$ whose union is $X$ has a connected intersection. A continuum is hereditarily unicoherent if each of its subcontinua is unicoherent.

Corollary 4. If $X$ is a homogeneous, decomposable continuum that is not aposyndetic, then $H^{1}(X) \neq 0$, and $H^{q}(X)=0$ for $q>1$.

Proof. Since $f^{*}: H^{q}(Y) \rightarrow H^{q}(X)$ is an isomorphism, it suffices to show these claims for the cohomology groups of $Y$. The second claim is true because $Y$ is onedimensional. To prove the first claim, recall [R2, Theorem 1, p. 450] that an acyclic one-dimensional continuum is hereditarily unicoherent. Jones [J2] has shown that a hereditarily unicoherent, homogeneous continuum is indecomposable.

There is a theorem for indecomposable, homogeneous continua that corresponds to the Aposyndetic Decomposition Theorem. It is called the Terminal Decomposition Theorem [R3], and it goes like this.

Theorem 5. If $X$ is a homogeneous, indecomposable continuum that contains a nondegenerate, proper, terminal subcontinuum and if $H^{1}(X) \neq 0$, then $X$ admits a continuous decomposition into mutually homeomorphic, indecomposable, homogeneous continua such that the quotient space $Y$ is a homogeneous, indecomposable continuum that contains no proper, nondegenerate, terminal subcontinuum.

The condition of nontrivial first cohomology of $X$ is necessary to insure the existence of maximal terminal, proper subcontinua of $X$; these are the elements of the decomposition. As before, the elements of the decomposition are cell-like continua of the same dimension as $X$. Hence, by the same proof as Theorem 3, we have the following theorem.

Theorem 6. If $X$ satisfies the hypotheses of Theorem 5, then the quotient space $Y$ of the terminal decomposition is one-dimensional.

Question 7. If $X$ satisfies the hypotheses of Theorem 5, is the quotient space $Y$ of the terminal decomposition of $X$ a solenoid? 
According to a theorem of Pavel Krupski [Kru, Theorem 3.1, p. 167], the answer is yes, provided that the complement of any subcontinuum of $Y$ has finitely many components.

\section{REFERENCES}

[B] R. H. Bing, Higher dimensional hereditarily indecomposable continua., Trans. Amer. Math. Soc. 21 (1951), 267-273. MR 13:265c

[HW] W. Hurewicz and J. Wallman, Dimension Theory, Princeton Univ. Press, Princeton, N.J., 1948.

[J1] F. Burton Jones, The aposyndetic decomposition of homogeneous continua, Topology Proc. 8 (1983), 51-54. MR 85g:54023

[J2] F. Burton Jones, Certain homogeneous unicoherent indecomposable continua, Proc. Amer. Math. Soc. 2 (1951), 855-859. MR 13:573a

[Kra] J. Krasinkiewicz, On mappings with hereditarily indecomposable fibers, Bull. Polish Acad. Sci. Math. 44 (1996), 147-156. MR 97g:54016

[Kru] P. Krupski, The disjoint arcs property for homogeneous curves, Fund. Math. 146 (1995), 159-169. MR 96a:54049

[L] M. Levin, Bing maps and finite dimensional maps, Fund. Math. 151 (1996), 47-52. MR 97e:54031

[N] S. B. Nadler, Jr., Continuum Theory, Marcel Dekker, Inc. New York, 1992. MR 93m:54002

[R1] J. T. Rogers, Jr., Cell-like decompositions of homogeneous continua, Proc. Amer. Math. Soc. 87 (1983), 375-377. MR 84e:54040

[R2] J. T. Rogers, Jr., Completely regular mappings and homogeneous aposyndetic continua, Canad. J. Math. 33 (1981), 450-453. MR 83a:54012

[R3] J. T. Rogers, Jr., Decompositions of continua over the hyperbolic plane, Trans. Amer. Math. Soc. 308 (1988), 277-291. MR 90a:54091

[R4] J. T. Rogers, Jr., Homogeneous continua, Topology Proc. 8 (1983), 213-233.

[R5] J. T. Rogers, Jr., Homogeneous hereditarily indecomposable continua are tree-like, Houston J. Math. 8 (1982), 421-428. MR 84b:54072

[R6] J. T. Rogers, Jr., Orbits of higher-dimensional, hereditarily indecomposable continua, Proc. Amer. Math. Soc. 95 (1985), 483-486. MR 86k:54054

[S] E. H. Spanier, Algebraic topology, McGraw-Hill Book Co., New York, 1966.

[W] J. J. Walsh, Dimension, cohomological dimension, and cell-like mappings. In "Shape Theory and Geometric Topology" (S. Mardesic, and J. Segal, eds.), Lecture Notes in Math, No. 870, Springer-Verlag, Berlin and New York, 1981, 105-118. MR 83a:57021

Department of Mathematics, Tulane University, New Orleans, Louisiana 70118

E-mail address: jim@math.tulane.edu 